\title{
Myelinaufbau schützt Nerven
}

\section{Neue Ansätze zur Remyelinisierung sollen bleibende Behinderungen bei MS-Kranken verhindern.}

Bei fortgeschrittener und progredienter MS schafft es der Körper immer weniger, die Schäden zu beheben, die nach dem Angriff des Immunsystems auf die Axonisolierung entstehen. Dringend nötig wären daher Therapien zum Myelinaufbau. Einige Ansätze wurden in diesem Jahr beim Kongress der American Academy of Neurology in Washington D.C. vorgestellt.

So prüften Forscher in der Studie RENEW mit 82 Patienten einen Antikörper gegen das Oberflächenprotein LINGO-1 bei Patienten mit einer akuten
Optikusneuritis, wie sie bei einem MSSchub auftreten kann. Die Blockade von LINGO-1 soll die Myelinbildung beschleunigen. Tatsächlich erhöhte sich die Leitfähigkeit der betroffenen Sehnerven unter dem Antikörper signifikant stärker als unter Placebo.

Ähnlich erfolgreich verlief eine Studie mit dem Antikonvulsivum Phenytoin. Die Substanz hemmt spannungsabhängige Natriumkanäle und erwies sich in Tierexperimenten als neuroprotektiv. Bei 86 Patienten mit Optikusneuritis wurden die Dicke der retinalen Nervenfaserschicht und das Makulavolumen im Vergleich zu Placebo deutlich gesteigert.

www.aerztezeitung.de

\section{ÜBRIGENS}

... bleiben $\mathbf{9 0 \%}$ der Patienten mit hochaktiver MS nach einer Stammzellentransplantation über drei Jahre hinweg schubfrei. Bei dem Verfahren ist die Rate schwerer Nebenwirkungen jedoch sehr hoch.

JAMA Neurol. 2015; 72(2): 159-169)

... verkürzt eine MS das Leben um etwa sieben Jahre. Laut einer kanadischen Analyse starben MS-Patienten im Schnitt mit 75,9 Jahren, solche ohne MS mit 83,4 Jahren.

Neurology 2015; online 27.Mai

... reduziert Kaffee möglicherweise das MS-Risiko. Nach Daten von zwei Fall-Kontroll-Studien scheint das Erkrankungsrisiko bei mindestens sechs Tassen täglich um etwa ein Drittel geringer zu sein.

Neurology 2015; 84(14) 545.004

\section{Häufige Symptome bei progredienter MS}

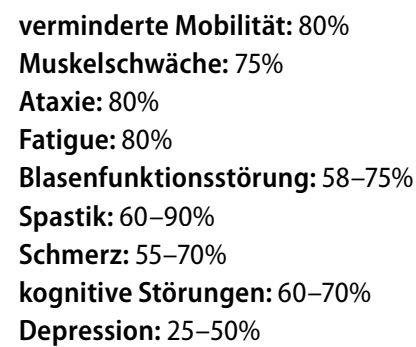

- Fatigue: Offenbar scheint körperliches Training eine Fatigue zu lindern, verhaltenstherapeutische Maßnahmen können nach Studiendaten ebenfalls helfen. Ermutigend sind auch Versuche zur medikamentösen Therapie mit Rivastigmin oder Diaminopyridin: Verarbeitungsgeschwindigkeit und motorische Aktivität ließen sich damit verbessern. Vitamin-DDerivate wie Alfacalcidol scheinen ebenfalls vielversprechend.

Blasenfunktionsstörungen: $\mathrm{Zu}$ Beginn einer Blasenüberaktivität werden häufig Anticholinergika oder antimuskarinerge Wirkstoffe eingesetzt. Auch Botulinumtoxin-Injektionen sind eine Option. In
Studien ließen sich damit Inkontinenzepisoden deutlich reduzieren - bei schubförmiger sowie auch bei progredienter MS. Bei leichten Störungen ist offenbar auch Beckenbodentraining effektiv.

Kognitive Störungen: Medikamentenstudien lieferten sehr uneinheitliche Ergebnisse, am ehesten waren Erfolge bei Substanzen zur Aufmerksamkeitssteigerung zu beobachten, etwa mit Methylphenidat, L-Amphetamin oder Modafinil, weniger mit klassischen Antidementiva. In einigen Studien wurden auch mit einem kognitiven Training Verbesserungen beobachtet, vor allem mit Methoden, die Vorstellungsvermögen und Kontext berücksichtigen.

Schmerzen: Kopf- und Gliederschmerzen sowie eine Spastik setzen den meisten $\mathrm{Pa}$ tienten mit progredienter MS zu. Neben dem üblichen Arsenal an Schmerzmitteln haben sich auch Cannabis-Präparate, Antidepressiva und Antiepileptika als wirksam erwiesen. In Studien mit Laufbandund Gehtraining gingen als Nebeneffekt auch die Schmerzen zurück. (Thomas Müller)

Lancet Neurol 2015; 14: 194-207

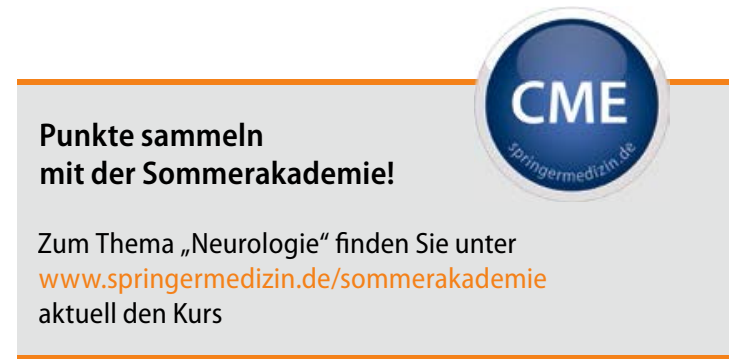

"Sensorische, motorische und autonome

Störungen: Das komplexe regionale

Schmerzsyndrom"

Mit e.Med teilnehmen

Mit e.Med haben Sie nicht nur Zugriff auf die aktuelle Sommerakademie, Sie können das komplette Angebot von Springer Medizin nutzen - zusätzlich erhalten Sie eine gedruckte Fachzeitschrift Ihrer Wahl. Mit rund 1600 Fortbildungskursen, davon über 500 CME-zertifiziert, ist die e.Akademie Deutschlands erste Adresse für Online-CME-Fortbildung.

Nutzen Sie die Sommerzeit, um reichlich CME-Punkte zu sammeln!

30 Tage kostenlos

Unser Kundenservice steht Ihnen wochentags (10-17 Uhr) kostenlos zur Verfügung: Tel. 08007780777 kundenservice@springermedizin.de 